IAC-06-A3.5.8

\title{
A COMPARATIVE ASSESSMENT OF DIFFERENT DEVIATION STRATEGIES FOR DANGEROUS NEO
}

\author{
Camilla Colombo, Joan Pau S. Cuartielles, Massimiliano Vasile, Gianmarco Radice \\ University of Glasgow, United Kingdom \\ c.colombo@aero.gla.ac.uk, p.sanchez@aero.gla.ac.uk,m.vasile@aero.gla.ac.uk, \\ g.radice@aero.gla.ac.uk
}

\begin{abstract}
In this paper a number of deviation strategies for dangerous Near Earth Objects (NEO) have been compared. For each strategy (i.e. Solar Collector, Nuclear Blast, Kinetic Impactor, Low-thrust Propulsion, Mass Driver) a multicriteria optimisation method has been used to reconstruct the set of Pareto optimal solutions minimising the mass of the spacecraft and the warning time, and maximising the deviation. Then, a dominance criterion has been defined and used to compare all the Pareto sets. The achievable deviation at the MOID, either for a low-thrust or for an impulsive variation of the orbit of the NEO, has been computed through a set of analytical formulas. The variation of the orbit of the NEO has been estimated through a deviation action model that takes into account the wet mass of the spacecraft at the Earth. Finally the technology readiness level of each strategy has been used to compute a more realistic value for the required warning time.
\end{abstract}

\section{INTRODUCTION}

Among the vast amount of small celestial bodies that orbits the Sun, Near Earth Objects (NEO) are those asteroids whose orbits allow them to get into Earth's neighbourhood. Apart from scientific reasons, such as the extensive amount of knowledge about formation, composition and evolution of the solar system, NEO represent a possible risk to mankind, even if not in a near term.

On average, every 26-30 million years a $10-\mathrm{km}$ sized asteroid strikes the Earth, while every 100 years a Tunguska class (100 $\mathrm{m}$ in size) asteroid hits. Each of these impacts permanently alters the characteristics of our planet to varying degrees. These events, and risks associated to our fragile ecosystem, have made the space community turn their attention to NEO [1]. Moreover advances in orbit determination of asteroids and theoretical studies on hazard characterization have increased the capability of predicting potential impacts.
Don Quijote [2], Deep Impact [3], NEAR [4] and ROSETTA [5], all of them space missions to asteroids or comets, evidence the interest of the scientific community, both in order to improve the current knowledge about the solar system and to test the capability to mitigate a dangerous asteroid if needed.

During these last few decades many deviation methods involving numerous physical interactions with the asteroid have been analysed. These strategies can be organized in four main groups: kinetic impactors, i.e. methods modelled as inelastic impacts [6][7]; attached propulsion devices [8] (e.g. electric/chemical engines, solar sails); induced thermooptical changes on the asteroid surface [9] (e.g. induced Yarkovsky effect, paints) and ablation methods [10] (e.g. lasers, solar mirrors). More recently, other advanced concepts have been considered such as gravitational tractors [11] and technologies that act on the magnetic field or the rotational properties of the asteroid.

Only a few authors have performed a partial comparative assessment of the numerous proposed mitigation strategies. Some of these emphasise a 
classification system based on NEO/spacecraft coupling [12], other systems are based on technology readiness and a third category on time to impact and/or intervention of the asteroid [13].

This paper presents a more exhaustive comparison of deflection methods according to different criteria. After a taxonomical classification of NEO physical characteristics (i.e. size, mass, shape and spin properties), composition (i.e. surface composition and heterogeneity, gas and dust emission) and orbital parameters (including inclination and eccentricity), the principal mitigation strategies have been evaluated in terms of several figures of merit: achieved miss distance at the Earth, anticipating time, total mass into orbit and technology readiness (estimated time to develop the required technology).

The miss distance between the asteroid and the Earth is the displacement of the position of an asteroid at the Minimum Orbit Interception Distance (MOID) achieved by a deviation manoeuvre applied before the encounter, while the anticipating time has been defined as the time difference between the impact epoch and the time a given strategy is applied.

Finally the paper presents a multi-criteria optimisation which provides a relative measure of the effectiveness of the different mitigation approaches.

\section{DEVIATION FORMULAS}

In order to study the effect of a general deflection strategy, we propose a set of analytical formulas that gives the variation of the MOID as a function of a variation of the orbit of the asteroid. This formulation is a generalization of what proposed by Carusi et al. [16], Scheeres et al. [17] and Izzo et al. [14][15], and yields results comparable with the results obtained by Conway [18] and Ross et al. [19].

Previous deflection formulas [14][15] where based on the modification of the orbital period due to a force acting on the asteroid. Only the effect on the orbital mean motion due to a change in the orbital energy was considered and other changes in the orbital geometry were neglected. As a consequence the resulting deviation could be maximised only by applying some action in a direction parallel to the velocity vector of the asteroid. Any other strategy producing an action in any normal direction could not be studied.

A more general approach was used by Conway to determine the near-optimal direction in which an impulsive manoeuvre should be given. In this case, the modified orbit of the asteroid was propagated analytically forward in time by means of Lagrange coefficients expressed through universal formulas. A similar approach was used by Park and Ross [19], who; in a subsequent work [20] studied also the influence of the gravitational effects of the Earth. Finally Carusi et al. presented the first n-body analysis, by means of a numerical procedure, but restricted the deflection manoeuvre to be applied along-track only.

In this work, the miss distance achieved with a given deviation action has been computed by means of proximal motion equations [21] expressed as a function of the orbital elements. The variation of the orbital parameters has been computed with Gauss' equations [22]. Proximal motion equations, already available in the literature for formation flying on general elliptical orbits, provide, with very little modifications, a simple and general mean to compute, with a good accuracy, the variation of the MOID. An analytical expression was derived in the case the spacecraft-asteroid interaction can be modelled as an impulsive manoeuvre.

Furthermore, the proposed analytical formulation can be used to compute the optimal direction of the deviating impulse and therefore the required $\delta \mathbf{v}$ to be imparted to the NEO for a given required variation of the MOID.

When the deviation action is modelled as a lowthrust action, instead, Gauss' equations can be numerically integrated to compute the variation of the orbital elements. The developed model has general validity and can be applied to every deviation strategy unless the deviated orbit is too distant from the original one.

Given the time of interception $t_{d}$ of a generic NEO, the objective is to calculate the deviation achieved at the Minimum Orbit Interception Distance from the Earth, by applying a deviation action. The effect on the asteroid of all the analysed deviation strategies has been modelled either as an impulsive $\delta \mathbf{v}$ at the interception time $t_{d}$ or as a continuous acceleration over an interval $\left[\begin{array}{ll}t_{d} & t_{e}\end{array}\right]$. These two cases have been described by different models, based on the same approach in order to compute the resulting change in MOID.

\section{Impulsive action deviation formulas}

The impulsive manoeuvre $\delta \mathbf{v}$ at time $t_{d}$ acts as a perturbation on the orbit of the NEO. The new orbit of the NEO can be considered to be proximal to the unperturbed one. If $\theta$ is the true anomaly of the NEO at the MOID along the unperturbed orbit and $\theta^{*}=\theta+\omega$ is the corresponding latitude, we can write the variation of the position of the NEO, after deviation, with respect to its unperturbed position by using proximal motion equations [21]: 


$$
\begin{aligned}
\delta s_{r} \approx & \frac{r}{a} \delta a+\frac{a e \sin \theta}{\eta} \delta M-a \cos \theta \delta e \\
\delta s_{\theta} \approx & \frac{r}{\eta^{3}}(1+e \cos \theta)^{2} \delta M+r \delta \omega+ \\
& +\frac{r \sin \theta}{\eta^{2}}(2+e \cos \theta) \delta e+r \cos i \delta \Omega \\
\delta s_{h} \approx & r\left(\sin \theta^{*} \delta i-\cos \theta^{*} \sin i \delta \Omega\right)
\end{aligned}
$$

where $\delta s_{r}, \delta s_{\theta}, \delta s_{h}$ are the displacements in the radial, transversal and perpendicular-to-the-orbit-plane directions respectively, so that $\delta \mathbf{r}=\left[\begin{array}{lll}\delta s_{r} & \delta s_{\vartheta} & \delta s_{h}\end{array}\right]^{T}$ and $\eta=\sqrt{1-e^{2}}$. The symbol $\delta$ is used to define the relative orbit in terms of difference between the nominal orbit and the perturbed one. The variation of the orbital parameters $a, e, i, \omega$ and $\Omega$ are computed through Gauss' planetary equations [22] considering an instantaneous change in the NEO velocity vector $\delta \mathbf{v}=\left[\begin{array}{lll}\delta v_{t} & \delta v_{n} & \delta v_{h}\end{array}\right]^{T}$ :

$$
\begin{aligned}
\delta a= & \frac{2 a^{2} v}{\mu} \delta v_{t} \\
\delta e= & \frac{1}{v}\left[2(e+\cos \theta) \delta v_{t}-\frac{r}{a} \sin \theta \delta v_{n}\right] \\
\delta i= & \frac{r \cos \theta^{*}}{h} \delta v_{h} \\
\delta \Omega= & \frac{r \sin \theta^{*}}{h \sin i} \delta v_{h} \\
\delta \omega= & \frac{1}{e v}\left[2 \sin \theta \delta v_{t}+\left(2 e+\frac{r}{a} \cos \theta\right) \delta v_{n}\right]+ \\
& -\frac{r \sin \theta^{*} \cos i}{h \sin i} \delta v_{h} \\
\delta M_{t_{d}} & =-\frac{b}{e a v}\left[2\left(1+\frac{e^{2} r}{p}\right) \sin \theta \delta v_{t}+\frac{r}{a} \cos \theta \delta v_{n}\right]
\end{aligned}
$$

The Gauss' equations have been written in a tangential-normal-binormal reference frame, so that $\delta v_{t}$ and $\delta v_{n}$ are the components of the impulsive deviation manoeuvre, in the plane of the osculation orbit, along the velocity vector and perpendicular to it. The variation $\delta M_{t_{d}}$ takes into account only the instantaneous change of the orbit geometry at time $t_{d}$. On the other hand due to the change in the semi-major axis, there is a variation of the mean motion $n$ with the associated variation in the mean anomaly:

$$
\delta M_{n}=\delta n\left(t_{M O I D}-t_{d}\right)=\delta n \Delta t
$$

where $t_{M O I D}$ is the time at the MOID along the orbit of the NEO and $\delta n=\sqrt{\frac{\mu}{a^{3}}}-\sqrt{\frac{\mu}{(a+\delta a)^{3}}}$. Eq. (2.3) takes into account the phase shifting between the Earth and the NEO. The total variation in the mean anomaly between the unperturbed and the proximal orbit is therefore:

$$
\begin{aligned}
\delta M= & -\frac{b}{e a v}\left[2\left(1+\frac{e^{2} r}{p}\right) \sin \theta \delta v_{t}+\frac{r}{a} \cos \theta \delta v_{n}\right]+ \\
& +\delta n \Delta t
\end{aligned}
$$

The result in Eq. (2.4) can be demonstrated as follows. Let's define $\tilde{M}_{M O I D}$ as the mean anomaly of the perturbed orbit at the MOID and $M_{M O I D}$ the mean anomaly of the nominal orbit at the MOID:

$$
\begin{aligned}
\tilde{M}_{\text {MOID }} & =M\left(t_{d}\right)+(n+\delta n)\left(t_{M O I D}-t_{d}\right)+\delta M_{t_{d}} \\
& =n\left(t_{d}-t_{0}\right)+(n+\delta n)\left(t_{M O I D}-t_{d}\right)+\delta M_{t_{d}} \\
M_{M O I D} & =n\left(t_{M O I D}-t_{0}\right) \\
\text { then } & \\
\delta M & =\tilde{M}_{M O I D}-M_{M O I D}=\delta n\left(t_{M O I D}-t_{d}\right)+\delta M_{t_{d}}
\end{aligned}
$$

Notice equations (2.1) are an approximation of the first order and thus they can be used until the relative orbit radius $\delta \mathbf{r}$ is small compared to the nominal orbit radius $\mathbf{r}$.

The accuracy of Eqs. (2.1) has been assessed by numerically propagating forward in time the deviated orbit of the asteroid and comparing the obtained variation in the position vector with the one predicted by Eqs. (2.1). The nominal trajectory has been propagated from the deviation point up to the MOID, in the interval $\left[\begin{array}{ll}t_{d} & t_{M O I D}\end{array}\right]$ and the deviated one has been integrated starting from the deviation point on the NEO orbit with the modified velocity vector $\mathbf{v}+\delta \mathbf{v}$. As a measure of accuracy we used the relative error between the variation in position computed numerically and analytically:

$$
e_{r}=\frac{\left\|\delta \mathbf{r}_{\text {propagated }}-\delta \mathbf{r}_{\text {estimated }}\right\|}{\left\|\delta \mathbf{r}_{\text {propagated }}\right\|}
$$

Figure 2.1 and Figure 2.2 show the relative errors as a function of the anticipating time $t_{M O I D}-t_{d}$ and $\delta \mathbf{v}_{t}$ along track (i.e. along the instantaneous velocity vector of the NEO). The relative error has been calculated for the asteroid 2000SG344 and for the asteroid 1979XB. These two different kinds of asteroid orbits, the former with $e<0.1$ and $i<10^{\circ}$, the latter with $e>0.1$ and $i>10^{\circ}$, have been chosen in order to study the influence of the orbital parameters on the accuracy of the deflection formulas. 


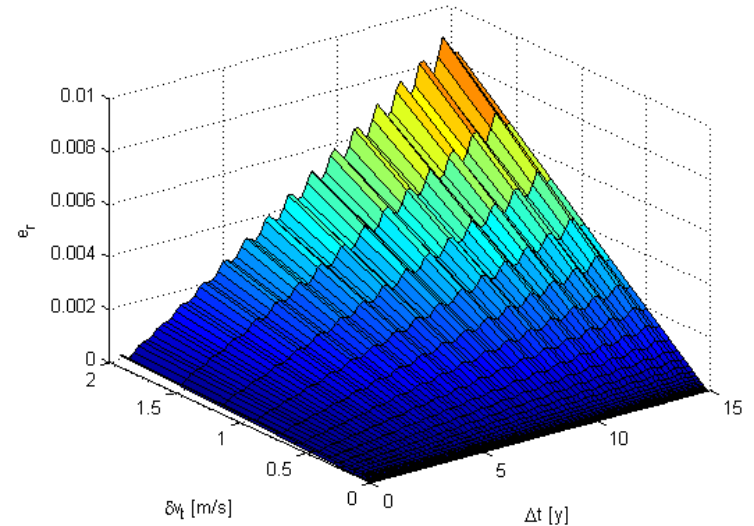

Figure 2.1: Relative error for 2000SG344 deviation.

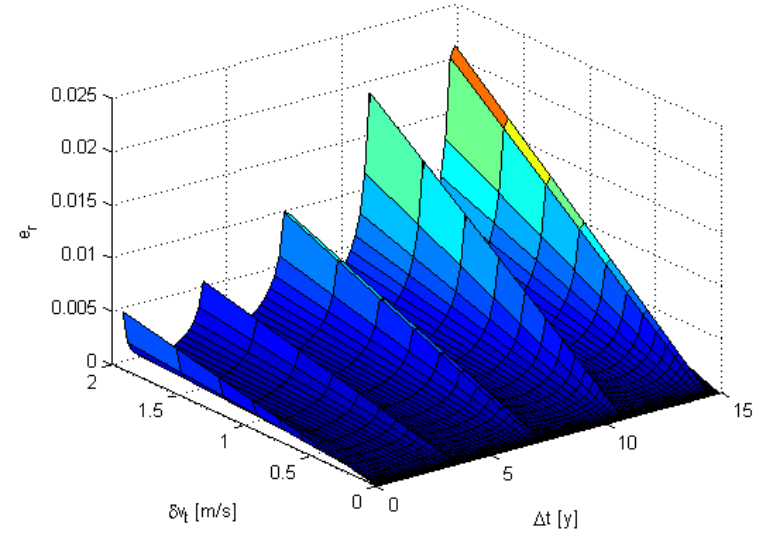

Figure 2.2: Relative error for $1979 \mathrm{XB}$ deviation.

Now if $\Delta \mathbf{r}=\left[\begin{array}{lll}\Delta s_{r} & \Delta s_{\theta} & \Delta s_{h}\end{array}\right]^{T}$ is the vector distance of the asteroid from the Earth at the MOID, and $\delta \mathbf{r}=\left[\begin{array}{lll}\delta s_{r} & \delta s_{\theta} & \delta s_{h}\end{array}\right]^{T}$ is the variation given by Eqs. (2.1), then the objective function for the maximum deviation problem is the following:

$$
J=\left(\Delta s_{r}+\delta s_{r}\right)^{2}+\left(\Delta s_{\theta}+\delta s_{\theta}\right)^{2}+\left(\Delta s_{h}+\delta s_{h}\right)^{2}(2.7)
$$

Notice that the objective function in Eq. (2.7) requires intrinsically to produce a variation that increases the MOID.

A maximum deviation analysis has been carried out to find the optimal direction of an impulsive manoeuvre to be given to the asteroid at a specific time in order to achieve the maximum deviation at the MOID.

\section{Low-thrust action deviation formulas}

When a continuous deviation action is applied, the total variation in orbital parameters has been computed by integrating Gauss' variational equations on the interval $\left[\begin{array}{ll}t_{d} & t_{e}\end{array}\right]$ :

$$
\begin{aligned}
\frac{d a}{d t}= & \frac{2 a^{2} v}{\mu} a_{t} \\
\frac{d e}{d t}= & \frac{1}{v}\left[2(e+\cos \theta) a_{t}-\frac{r}{a} \sin \theta a_{n}\right] \\
\frac{d i}{d t}= & \frac{r \cos \theta^{*}}{h} a_{h} \\
\frac{d \Omega}{d t}= & \frac{r \sin \theta^{*}}{h \sin i} a_{h} \\
\frac{d \omega}{d t}= & \frac{1}{e v}\left[2 \sin \theta a_{t}+\left(2 e+\frac{r}{a} \cos \theta\right) a_{n}\right]+ \\
& -\frac{r \sin \theta^{*} \cos i}{h \sin i} a_{h} \\
\frac{d M}{d t}= & n-\frac{b}{e a v}\left[2\left(1+\frac{e^{2} r}{p}\right) \sin \theta a_{t}+\frac{r}{a} \cos \theta a_{n}\right]
\end{aligned}
$$

where $\mathbf{a}=\left[\begin{array}{lll}a_{t} & a_{n} & a_{h}\end{array}\right]^{T}$ is the acceleration function of time, given by the low-thrust strategy. Note than in this case the derivative of $M$, in the 6th equation of system (2.8), takes into account also the change of mean anomaly due to the change in the angular velocity $n$ on the thrusting arc, because the term $n$ can not be ignored as in the impulsive manoeuvre case. Called $\alpha(t)=\left[\begin{array}{llllll}a & e & i & \Omega & \omega & M\end{array}\right]^{T}$ the vector of the orbital parameters, we define:

$$
\Delta \mathbf{a}=\mathbf{a}\left(t_{d}\right)-\mathbf{a}\left(t_{e}\right)=\left[\begin{array}{llllll}
\Delta a & \Delta e & \Delta i & \Delta \Omega & \Delta \omega & \Delta M
\end{array}\right]^{T}
$$

the finite variation of the orbital parameters on the NEO perturbed orbit, in the interval $\left[\begin{array}{ll}t_{d} & t_{e}\end{array}\right]$, obtained form the numerical integration of Eqs.(2.8).

We can note that $\delta a=\Delta a, \delta e=\Delta e, \delta i=\Delta i$, $\delta \Omega=\Delta \Omega, \delta \omega=\Delta \omega$, to be substitute in the proximal motion equations, while $\delta M$ has to be computed in a way analogous to (2.4). Since:

$$
\begin{aligned}
\tilde{M}_{M O I D}= & M\left(t_{e}\right)+n_{f}\left(t_{M O I D}-t_{e}\right)= \\
& M\left(t_{d}\right)+\Delta M+n_{e}\left(t_{M O I D}-t_{e}\right)= \\
& n\left(t_{d}-t_{0}\right)+\Delta M+n_{e}\left(t_{M O I D}-t_{e}\right) \\
M_{M O I D}= & n\left(t_{M O I D}-t_{0}\right)
\end{aligned}
$$

we can conclude that:

$$
\begin{aligned}
\delta M= & \tilde{M}_{M O I D}-M_{M O I D}= \\
& \left(n_{e}-n\right) t_{M O I D}+n t_{d}-n_{e} t_{e}+\Delta M
\end{aligned}
$$

Where $n$ is the nominal angular velocity and $n_{e}-n=\Delta n$. At this point Eqs. (2.1) can be used again to compute the consequent $\delta \mathbf{r}$. 


\section{ACTION MODELS}

In order to evaluate the performance achievable with each deviation strategy, a set of simple models has been developed. Each model yields the total impulse imparted to the asteroid and the total required spacecraft mass. The general analytical form of all the models is:

$$
\begin{aligned}
& \Delta \mathbf{v}=f\left(m_{d}, I d_{N E O}\right) \\
& \text { Thrust }=f\left(m_{d}, I d_{N E O}\right)
\end{aligned}
$$

where $m_{d}$ is dry mass of the spacecraft, which is defined as the mass available to alter the trajectory of the celestial body after the transfer from Earth to the asteroid, and $I d_{N E O}$ is the asteroid identification number.

\section{Solar Collector}

The so-called solar collector is supposed to be an inflatable mirror that focuses enough energy onto the surface to ablate it. The material evaporated produces a plume of gas, which provides a constant thrust.

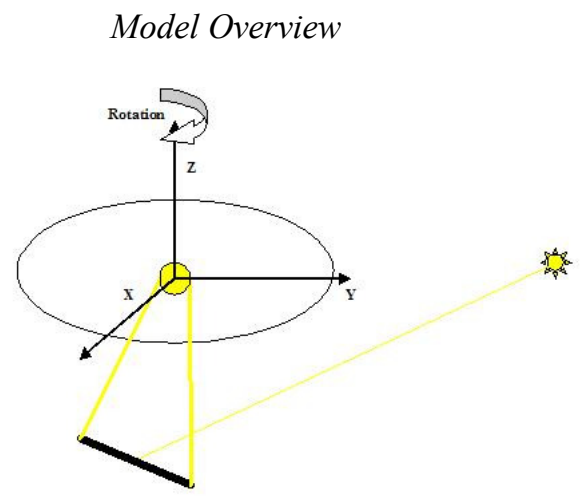

Figure 3.1: $\quad$ Model Outline. Sun reflexed in a mirror is focused into the asteroid equator.

A beam spot (see Figure 3.1), with enough energy density to trigger the evaporation, is focused onto the surface of the asteroid. The time a portion of the surface spends in the spot beam is a function of the angular rotation of the asteroid and of the size of the spot. A key parameter, defining the efficiency of the system, is the so-called focusing capability, which is defined as the ratio between the aperture area of the mirror and the area of the illuminated spot on the surface.

The model assumes the system to be an infinite long rod, with the illuminated spot in one side of it. The illuminated surface is at a temperature of $1800 \mathrm{~K}$, which is the sublimation temperature of the forsterite [23]. It is easy to proof that the conduction loss trough the perimeter of this rod is much smaller than the energy loss because of the movement of the surface (asteroid rotation and fixed beam), for this reason it is believed that the infinite long rod is a good approximation to the system [24].

Sublimation is due to the total absorbed energy. The net absorbed energy is the total energy focused on the surface minus the radiation and conduction losses. The radiation loss is easily calculated through the blackbody radiation formula $\left(\dot{E}_{\text {radiation }}=\sigma \cdot \varepsilon \cdot T^{4}\right)$, while energy loss by conduction can be computed by solving the following differential equation:

$$
\frac{\partial^{2} T}{\partial x^{2}}=\frac{\rho c}{k} \frac{\partial T}{\partial t}
$$

which will provide us the temperature profile $(T)$ depending of the variables $x$ and time $t$. Eq. (3.1) is solved with the following initial and boundary conditions:

$$
\begin{aligned}
& T(x, 0)=T_{0}=278 \mathrm{~K} \\
& T(0, t)=T_{\text {ablation }}=1800 \mathrm{~K} \\
& \lim _{x \rightarrow \infty} T(x, t)=T_{0}=278 \mathrm{~K}
\end{aligned}
$$

We solved Eq. (3.1), using Laplace transformation and the expression derived is:

$$
T(x ; t)=278+1522 \cdot \operatorname{erfc}\left(\frac{x}{2 \sqrt{\alpha \cdot t}}\right)
$$

where $\alpha=\frac{k}{\rho c}$.

Finally, to calculate the conduction, the derivative of the temperature profile is calculated through a series expansion of the complementary error function $(\operatorname{erfc}(x))$, at that time the energy conduction in the surface $(x=0)$ function of time develops the following form:

$$
\dot{E}_{\text {conduction }}=\frac{1522}{\sqrt{\frac{\pi \cdot t}{\rho \cdot c \cdot k}}}
$$

Where $\rho$ is density, $c$ is heat capacity $(750 \mathrm{j} / \mathrm{kg} / \mathrm{k})$ and $K$ is thermal conductivity.

\section{Mass expelled integral}

In order to know the acceleration produced by the mass expelled, we need to know the mass evaporation flow and mean velocity of the gas. The overall system energy balance will help with the evaporation flow.

$$
E_{\text {in }}-E_{\text {out }}=H \cdot \frac{d m}{d t}
$$

Where $E_{\text {in }}-E_{\text {out }}$ is the energy power available for evaporation, $\mathrm{H}$ is the energy of sublimation and $d \mathrm{~m} / \mathrm{dt}$ 
is the mass per second that is being evaporated or sublimated.

Writing this equation with all its terms and isolating the derivative of the mass and time it is obtained:

$$
\frac{d m}{d t}(t)=\frac{1}{H}\left(\rho_{\text {power }}-\sigma \cdot \varepsilon \cdot T^{4}-\frac{1522}{\sqrt{\frac{\pi \cdot t}{\rho \cdot c \cdot k}}}\right)
$$

Where $\rho_{\text {power }}$ is the energy density incoming into the system, taking into account efficiency of the mirror and albedo of the asteroid.

In order to calculate the total mass sublimated we perform an integral of the surface under the illuminated spot, taking into account the relation between the $x$ coordinate and time of exposition. We must pay attention to the negative results of $d m / d t$, as this means simply that the evaporation is not taking place. Consequently the limits of the integration must be fixed in order of avoiding negative results. Finally, the integral to solve is:

$$
\frac{d m}{d t} \text { total }=\int_{x_{i n}}^{x_{\text {fin }}} \int_{Y_{\min }}^{Y_{\max }} \frac{d m}{d t}(t) \cdot d x \cdot d y
$$

The change of variables $x=V_{r o t} \cdot t$, so $d x=V_{r o t} \cdot d t$ has been performed, since the coordinate $x$ and the time of exposition are related through the surface velocity. As a result the integral has the following final shape:

$$
\begin{aligned}
& \frac{d m}{d t} \text { total }=2 \cdot V_{\text {rot }} \cdot \int_{t_{\text {in }}}^{\sqrt{\frac{D^{2}-4 Y^{2}}{\text { rot }^{2}}}} \int_{0}^{Y_{\max }}\left(A-B \cdot \sqrt{\frac{1}{t}}\right) \cdot d t \cdot d y \\
& A \equiv \frac{1}{H}\left(\rho_{\text {power }}-\sigma \cdot \varepsilon \cdot T^{4}\right) \\
& B \equiv \frac{1522}{H} \sqrt{\frac{\rho \cdot c \cdot k}{\pi}}
\end{aligned}
$$

\section{Final Thrust}

Once the evaporation flux is computed, the average velocity of the particles is determined using the Maxwell distribution for particles of an ideal gas.

$$
\bar{V}=\sqrt{\frac{8 K T}{\pi M}}
$$

where $K$ is the Boltzman constant, $T$ is the sublimation temperature $(1800 \mathrm{~K}$ from Wang et. al. [23]) and $M$ is the mass of a single molecule of $\mathrm{Mg}_{2} \mathrm{SiO}_{4}$. At that time the thrust can be calculated multiplying the mass flux, the average velocity and a scattering factor $(2 / \pi)$.

$$
\text { Thrust }=\frac{2}{\pi} \cdot \frac{d m}{d t} \text { total } \cdot \bar{V}
$$

This acceleration is supposed to be tangent to the trajectory. Afterwards this thrust will be introduced in Gauss' equations (2.8), along with the equations of asteroid mass consumption (3.2) on the surface due to sublimation, to obtain the total variation in orbital parameters over the interval $\left[\begin{array}{ll}t_{d} & t_{e}\end{array}\right]$.

\section{Spacecraft Sizing}

. The mass of the spacecraft is estimated from the aperture of the mirror by using a simple correlation formula [25]:

$$
D[\mathrm{~m}] \approx 2.155\left(m_{d}[\mathrm{~kg}]\right)^{2 / 3}
$$

We use this correlation formula to estimate the mass of the mirror which represents the payload of the mission. The payload mass is then assumed to account for the $50 \%$ of the total mass of the spacecraft.

\section{Electric Propulsion}

In this case the linear momentum of the asteroid is perturbed by a thrust given by a propulsion system attached to its surface.

\section{Spacecraft Sizing}

The system could consist of at least two engines situated in opposite spots along the equator of the asteroid. By scheduling properly the periods when the engines are switch on and off we can obtain a constant thrust and a limited scattering factor. The scattering factor takes into account the misalignment from the optimal thrusting direction. Assuming that the power available comes from a subsystem the weight of which is $50 \%$ of the dry mass and that the power obtainable is about $20 \mathrm{~W} / \mathrm{kg}$ [26], the thrust can be calculated using an averaged thrust/power ratio of the most common Ion thrusters [26], $34 \mathrm{mN} / \mathrm{kW}$. This hypothesis leads to the following relation:

$$
\text { Thrust }=s_{\text {Scattering }} \cdot 0.5 \cdot \frac{m_{d}}{2} \cdot \frac{1}{0.02[\mathrm{~kW} / \mathrm{kg}]} \cdot 0.034[\mathrm{~N} / \mathrm{kW}]
$$

Finally, the mass consumption or mass propellant is calculated by using the rocket equation with an $I_{s p}$ of $3080 \mathrm{~s}$. 


\section{Mass Driver}

The idea behind this strategy is to change the linear momentum of the asteroid by using some ejected asteroid material. This ejected material would be accelerated by a catapult or a gun system, able to convert the available spacecraft power into kinetic energy.

$$
K E=\frac{1}{2} m \cdot v_{e}^{2}
$$

Obviously the conversion of energy from electric to kinetic is far from being optimal, but we could suppose that between the power system and payload (i.e. catapult or gun) the spacecraft is able to convert $50 \%$ of the energy available into kinetic energy [27] (i.e. $50 \%$ of mechanical loss). The excess velocity of the expelled material and the mass expelled with each shot are two parameters that would come from the engineering design of the system. However, in the literature it is common to read that an optimal and realistic excess velocity would be in between 100 and $300 \mathrm{~m} / \mathrm{s}$ [27][28] from which expelled mass can be estimated.

Once the spacecraft has landed and the operations have started, it would dig and collect material during most of its operating time and shot this material when pointing within 5 degrees of the orbital tangential direction.

\section{Spacecraft Sizing}

In our case the $50 \%$ of the dry mass has been considered to be power system, as it needs to be maximized. We average the specific power of several power systems, since is not into the scope of this paper to discuss the best engineering design, but to compare methods. The ratio obtained is $20 \mathrm{~W} / \mathrm{kg}$ [26] and with this the power available is known. With the rotation period and the configuration described above is straightforward to calculate the time available to shot, consequently the energy is known, and since we have fixed the excess velocity to $200 \mathrm{~km} / \mathrm{s}$ the mass expelled can be worked out with Eq. (3.3).

$$
\begin{aligned}
& m_{\text {payload }}=(50 \%) \cdot m_{d} \\
& \text { Power }=20[\mathrm{~W} / \mathrm{kg}] \cdot m_{\text {payload }} \\
& m_{\text {launch }}=\frac{(50 \%) \cdot 2 \cdot \text { Power } \text { Period }_{\text {rotation }} \cdot\left(\frac{10^{\circ}}{360^{\circ}}\right)}{V_{e}^{2}}
\end{aligned}
$$

The change in the velocity of the asteroid is determined using a simple conservation of linear momentum and taking into account the change in the asteroid mass due to every shot.

$$
\delta \mathbf{v}=\frac{m_{\text {launch }}}{m_{N E O}(t)} v_{e}
$$

At each $\delta \mathbf{v}$ corresponds a finite variation in orbital elements and the new set of orbital parameters must be calculated before the subsequent impulsive action. The finite change in the true anomaly has been added to the Gauss' equations (2.2) as well:

$$
\delta \theta_{t_{d}}=-\frac{1}{e v}\left[2 \sin \theta \delta v_{t}+\left(2 e+\frac{r}{a} \cos \theta\right) \delta v_{n}\right]
$$

The mean anomaly before each mass ejection is computed by the first equation of (2.5) and from that the Kepler's equation is solved for the osculating eccentric anomaly and the true anomaly, by a Newton method described in [22].

\section{Nuclear Blast}

Despite the three possibilities related with nuclear technology (i.e. stand-off, surface or buried explosions), we just considered the stand-off explosions in this paper. The reason is because a nuclear explosion at a certain distance of the asteroid is relatively solid against the uncertainties on asteroid materials, components, shape, etc. Therefore, since the goal was to compare nuclear technology methods, the stand-off technique seemed a good candidate.

\section{Stand-off model}

The kinetic energy provided using this method is obtained using a radiative nuclear explosive. The device is detonated at a certain optimal distance from the asteroid. Considering a spherical model of the asteroid this distance would be $(\sqrt{2}-1) R$ [28], achieving the highest coupling in energies; 0.3 of the total radiative energy is delivered over a 0.3 of the asteroid's area. The radiation projected over the asteroid is absorbed by a thin layer of surface, the depth depends on the penetration of the nuclear radiation and the density of the asteroid, in our model it has been considered $20 \mathrm{~cm} \mathrm{[28].}$

Because of the sudden change in temperature, a tensile failure of the surface is expected. This phenomenon would expel the mass radiated with a certain velocity (44 $\mathrm{m} \mathrm{s}^{-1} \mathrm{kton}^{-1}$ [28]). Taking into account the scattering of the surface material, the lineal momentum provided to the asteroid is calculated.

\section{Spacecraft Sizing}

Thinking in the warhead carried as the payload of the spacecraft, we can suppose then that the $30 \%$ of 
the dry mass will be the nuclear device [26]. Knowing the mass of it, the energy available in the explosion is calculated using the ratio $0.75 \mathrm{ktons} \mathrm{kg}^{-1}[29][30]$.

$$
\begin{aligned}
& \text { Nuclear }_{\text {head }}=(30 \%) \cdot m_{d} \\
& \text { Energy }[\mathrm{ktons}]=0.75\left[\mathrm{ktons} \cdot \mathrm{kg}^{-1}\right] \cdot \text { Nuclear }_{\text {head }}
\end{aligned}
$$

The excess velocity is calculated next, using the following relation:

$$
V_{e}=0.3 \cdot 44\left[\mathrm{~m} \cdot \mathrm{s}^{-1} \cdot \mathrm{kton}^{-1}\right] \cdot \text { Energy }[\text { ktons }]
$$

The approximate ratio of asteroid blown off after the nuclear explosion would be:

$$
\text { Volume }=\frac{4 \pi \cdot R_{N E O}^{2} \cdot(0.3) \cdot(0.2[\mathrm{~cm}])}{\frac{4}{3} \pi \cdot R_{N E O}^{3}}
$$

To conclude, the increment in the asteroid velocity is computed using:

$$
\Delta v=\frac{\text { Volume } \cdot V_{e} \cdot s_{\text {Scattering }}}{(1-\text { Volume })}
$$

This resulting increment of velocity is performed in the direction of the final velocity of the transfer trajectory, which depends on the arrival and launch date.

\section{Kinetic Impactor}

The Kinetic Impactor is the simplest concept of asteroid hazard mitigation. Here the asteroid linear momentum is modified by the collision of a certain mass, the spacecraft. This collision is modelled as a simple inelastic collision with a certain momentum enhancement [6].

This factor takes into account that despite the momentum provided to the initial mass of the asteroid is only the calculated using the inelastic collision model, the momentum of the asteroid itself is enhanced by the blast of material expelled after the collision. In order to be conservative in our models, the value of the enhancement chosen is 2 , as at the light of Tedeschi's experiments seems that this value could be between 2 and 5 .

The variation of velocity imparted by the spacecraft (i.e. impactor) is therefore given by:

$$
\delta \mathbf{v}=\beta \frac{m_{S / C}}{\left(m_{N E O}+m_{S / C}\right)} \Delta \mathbf{v}_{S / C}
$$

Where $\beta$ is the momentum enhancement factor and the relative velocity of the spacecraft with respect to the asteroid at the deviation point $\Delta \mathbf{v}_{S / C}$ is computed from the solution of a Lambert's problem, consequently the direction of the $\delta \mathbf{v}$ provided depends on the arrival and launch date.

\section{TRANSFER TRAJECTORY}

The transfer path and the consequent propellant mass required have been determined with two techniques, depending of the propulsion system adopted. For a high-impulse system a two-impulse Lambert's solver has been used, while for a low-thrust trajectory a shape-based approach has been followed instead [31]. In this work for the kinetic impactor and the nuclear blast strategies a high-impulse trajectory has been selected with a specific impulse of 315 s, while for the other deviation methods a low-thrust transfer with specific impulse of $2500 \mathrm{~s}$ has been computed.

\section{OPTIMISATION PROCEDURE}

A multi-criteria optimisation allowed a comparison of different strategies. The search of optimal sets of optimisation parameters is performed by a global optimisation procedure based on an incremental branch and prune of the solution space, combined with an agent-based search technique [32].

A convenient representation of the optimal sets is the Pareto front constituted by all the sets that have higher fitness and lower cost than points corresponding to other choices of parameters and are said to be "dominated" by another point. According to the definition, a set of parameters is Pareto optimal if there exists no other feasible vector of variables which would decrease some criterion without causing a simultaneous increase in at least one other criterion. This definition gives not one unique solution, but a set of not-dominated solutions, that generate the Pareto front.

\section{Optimisation parameters}

The MOID between the Earth and the NEO orbits has been calculated and a certain date when the asteroid crosses this point has been selected. The Earth is not necessary in that point on the crossing time, because the aim of this model is to measure the achieved deviation not to reproduce a real impact scenario. The selected figures of merit of the optimisation procedure are:

- the warning time $t_{w}=t_{d}-t_{M O I D}$, defined as the interval between the launch date and the time at the MOID;

- the mass in space $m_{0}$ is the wet mass on the Earth orbit after launch;

- the total deviation $\Delta \mathbf{r}$ at the MOID.

In order to submit to the hypotheses of the proximal motion equation the relative orbit radius $\delta \mathbf{r}$ 
must be small compared with the nominal orbit radius $\mathbf{r}$, the maximum deviation accepted has been fixed as the Earth-Moon distance, being this one a sufficient deviation in order to avoid any impact.

\section{ANALYSIS}

In order to obtain a convenient set of data from which to perform not just a comparison of the different strategies but to contrast their performance with different NEO characteristics, it was decided to choose, as a first set, 6 asteroids from the three NEO categories (Apollo, Atens and Amor). These asteroids where chosen to be within 1 to $9 \times 10^{10} \mathrm{~kg}$ and 1 to $9 \mathrm{x}$ $10^{12} \mathrm{~kg}$ in mass (Table 6.1).

Table 6.1: Asteroids chosen for this analysis.

\begin{tabular}{|l|l|l|}
\hline NEO Category & $\mathbf{1 - 9}^{\mathbf{1 0}} \mathbf{1 0} \mathbf{k g}$ & $\mathbf{1 - 9}^{\mathbf{1}} \mathbf{1 0}^{\mathbf{1 2}} \mathbf{k g}$ \\
\hline Atens & Apophis & $1999 \mathrm{KW}$ \\
\hline Apollo & Itokawa & Castalia \\
\hline Amor & Quetzalcoatl & Nyx \\
\hline
\end{tabular}

The solutions for each of these asteroids can be summarized in a set of Pareto fronts, one for each strategy. In this paper just one Pareto front for each deviation method has been represented: Nuclear Impactor Pareto front for 19999KW4 in Figure 6.1, Solar Collector Pareto front for Nyx in Figure 6.2, Kinetic Impactor Pareto front for Apophis in Figure 6.3, Electric Propulsion Pareto front for Castalia in Figure 6.4, and Mass Driver Pareto front for Castalia in Figure 6.5. In fact, despite the physical and orbital differences among the NEO from Table 6.1, the shape of the Pareto fronts is most strongly defined by the mitigation strategy used.

NEO orbital characteristics, size and rotational period play as well an important role in modelling the surface of the Pareto front, sizing it and changing slightly the inclination and position in the three dimensional space formed by mass in space $\left(m_{0}\right)$, warning time $\left(t_{w}\right)$ and deviation $\delta \mathbf{r}$ at the MOID.

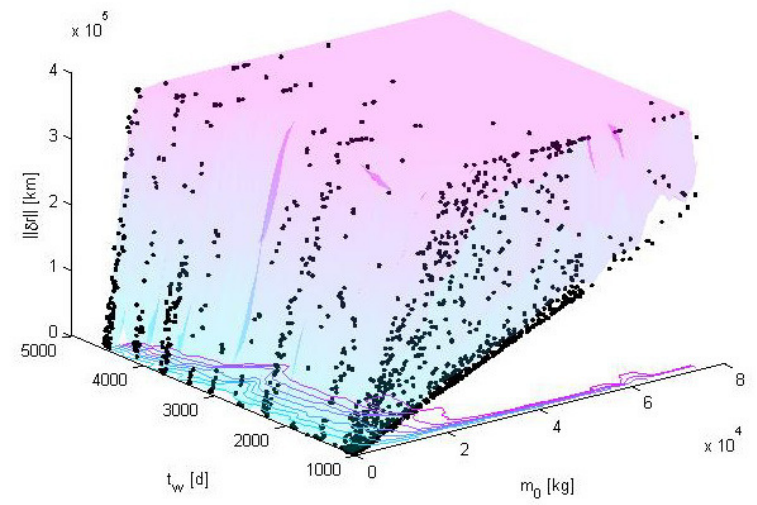

Figure 6.1: Nuclear Impactor Pareto front for 1999KW4.

As can be observed there are two common features in all the Pareto fronts. The former, the increase of deviation in $m_{0}$ (initial mass) dimension, with linear or quadratic behaviour, which is directly related with the analytic models developed for this experiment. The latter, the orbital period periodicity in the warning time $\left(t_{w}\right)$ dimension, directly related with the optimal moment during the orbit to apply the increment of velocity or to start to apply the acceleration. Note that for low thrust propulsion this periodicity of the orbit is only evident for short warning times, consistent with the fact that for long warning times the moment during the orbit chosen to start the impulse is less decisive. For most of the methods it is possible to make out the waves due to the asteroid orbit periodicity, although because of the dominance of the surface this shape has been reduced to steps.

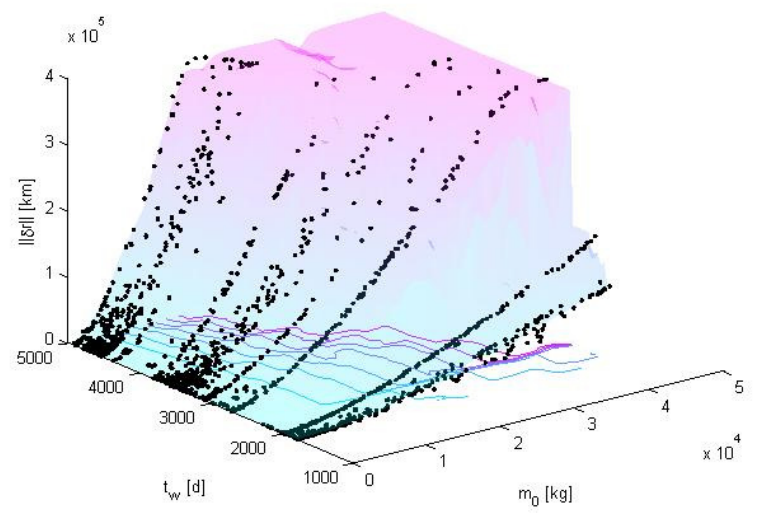

Figure 6.2: $\quad$ Solar Collector Pareto Front for Nyx.

Clearly, the two deviation methods that achieve the best results are Nuclear Impactor and Solar Collector. The plateau at the top of both Pareto fronts is due to the limit in deviation that it was chosen. This limit 
stops the program when the deviation reaches the Earth-Moon distance.

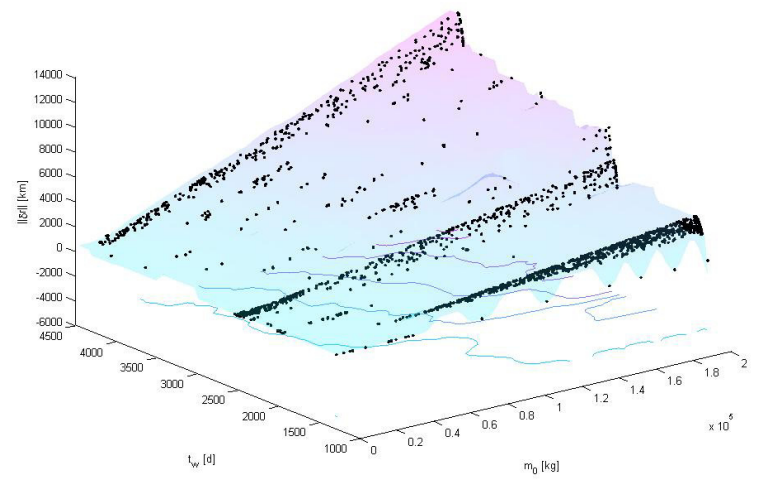

Figure 6.3: Kinetic Impactor Pareto front for Apophis.

Despite the Plateau for the Nuclear Impactor, Nuclear and Kinetic have similar functioning. Since the two methods are impulsive methods, the Pareto front is dominated by the periodicity of the asteroid.

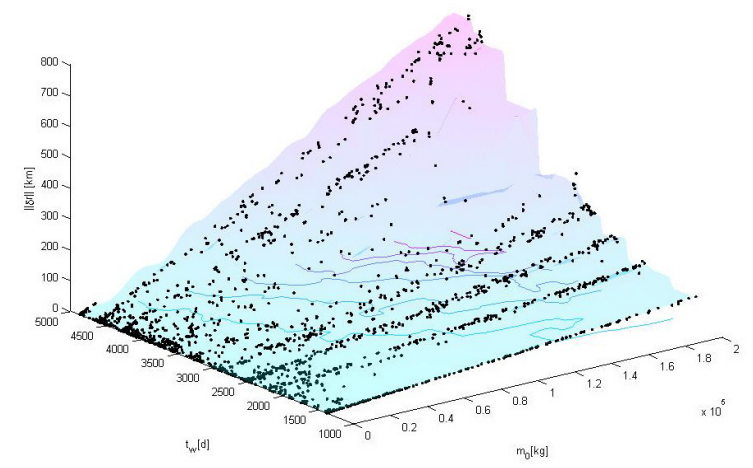

Figure 6.4: Electric Propulsion Pareto front for Castalia.

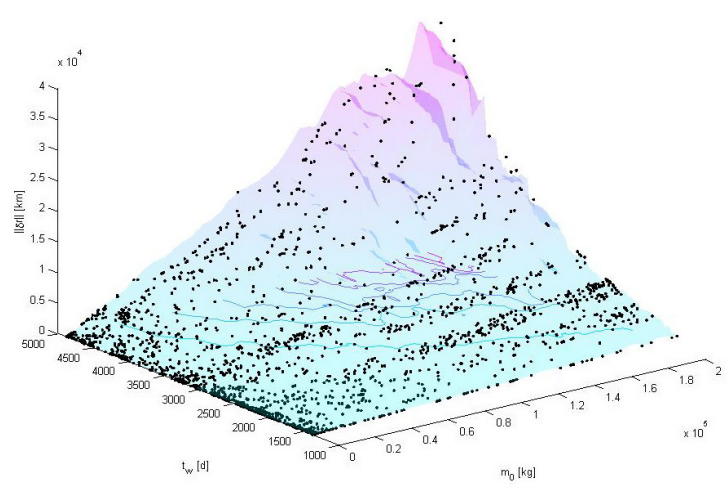

Figure 6.5: $\quad$ Mass Driver Pareto front for Castalia.

\section{MULTICRITERIA ANALYSIS}

The data of the different methods and asteroids has been compared studying its dominance.

A component $i$ of method $A$ is said to dominate a component $j$ of method $B$ if all the elements of $\mathbf{f}_{j}^{B}$ are smaller than all the elements of $\mathbf{f}_{i}^{A}$, where:

$$
\mathbf{f}_{i}^{A}=\left[f_{1, i}^{A}, f_{2, i}^{A}, \ldots, f_{k, i}^{A}\right]^{T}
$$

The dominance of an element $i$ of method $A$ with respect to method $B$ is the cardinality of the set of dominated components.

$$
I_{i}\left(m_{A}\right)=\left|\left\{j \mid \mathbf{f}_{i}^{A} \prec \mathbf{f}_{j}^{B}\right\}\right|
$$

Method $A$ dominates method $B$ if the sum of components of $A$ that are dominated by $B$ is less than the sum of the components of $B$ that are dominated by A.

$$
\begin{aligned}
& d_{i}=\left\{\begin{array}{l}
1 \text { if } I_{i}>0 \\
0 \text { if } I_{i}=0
\end{array}\right. \\
& m_{A} \succ m_{B} \Rightarrow \frac{1}{N_{A}} \sum_{i=1}^{N_{A}} d_{i}\left(m_{A}\right)<\frac{1}{N_{B}} \sum_{j=1}^{N_{B}} d_{j}\left(m_{B}\right)
\end{aligned}
$$

The dominance of the different methods is represented here in Table 7.1, Table 7.2 and Table 7.3. To understand the table we look at a certain column and check the value in a certain row. If this value is positive means that the method in the column

\begin{tabular}{|c|c|c|c|c|}
\hline & Nuke & Impactor & Solar & MD \\
\hline Impactor & 100 & & & \\
\hline Solar M. & 100 & -100 & & \\
\hline MD & 100 & -100 & 86 & \\
\hline $\mathrm{LT}$ & 100 & -100 & 100 & 100 \\
\hline
\end{tabular}
dominates the method in the row, if it is negative means that the column method is dominated.

\begin{tabular}{|c|c|c|c|c|}
\hline & Nuke & Impactor & Solar & MD \\
\hline Impactor & 48 & & & \\
\hline Solar M. & 74 & -100 & & \\
\hline $\mathrm{MD}$ & 92 & -100 & 100 & \\
\hline $\mathrm{LT}$ & 88 & -100 & 100 & 100 \\
\hline
\end{tabular}

Table 7.1: $\quad$ Nyx Comparison table ( $\mathrm{a}=1.93$ A.U.)

\begin{tabular}{|c|c|c|c|c|}
\hline & Nuke & Impactor & Solar & MD \\
\hline Impactor & 99.9 & & & \\
\hline Solar M. & -32 & -100 & & \\
\hline MD & 99.9 & -100 & 100 & \\
\hline LT & 99.9 & -99 & 100 & 100 \\
\hline
\end{tabular}

Table 7.2: $\quad$ Castalia Comparison table ( $\mathrm{a}=1.06$ A.U.).

Table 7.3: $\quad$ 1999KW4 Comparison table ( $\mathrm{a}=0.64$ A.U.). 
The results in these tables are the difference between the approximate percentage of dominance; for example in Table 7.3 (i.e. 1999KW4) the nuclear method is dominated by the solar by a $34 \%$, which means that solar method dominates over nuclear in $68 \%$ of the domain, therefore the difference between Solar and Nuclear domination is $32 \%$.

If the sum of the two percentages is more than 100 it indicates that in some regions of the domain both methods have equal dominance.

For Nyx and Castalia, the results show a clear domination of the nuclear method, followed by Solar, Mass Driver, Low Thrust and Kinetic, in this order. While for 1999KW4, Solar Method dominates over the others, followed by Nuclear, Mass Driver, Low Thrust and Kinetic Impactor.

This difference between the Atens asteroid and the other two is due to the semi-major axis, since it is directly related with the solar flux.

We can observe the same behaviour with the three small asteroids. Again with Apophis we observe a dominance of the Solar Collector over the others.

\section{Technology Readiness Level}

As an additional criterion we consider the technology readiness level of each method as a measure of the expected reliability. We define a $T R L$ factor or $T R L f$ by mapping the scale from 1 to 8 (Table 7.4) into the interval [ $\left[\begin{array}{ll}0 & 1\end{array}\right]$, where $T R L f$ equal to 1 means full operational capability.

Table 7.4: Technology Readiness Levels.

\begin{tabular}{|c|c|}
\hline $\mathbf{1}$ & No development performed \\
\hline $\mathbf{2}$ & Conceptual design formulated \\
\hline $\mathbf{3}$ & Conceptual design tested analytically or experimentally \\
\hline $\mathbf{4}$ & Critical function/characteristic demonstrated \\
\hline $\mathbf{5}$ & Component or Breadboard tested in relevant environment \\
\hline $\mathbf{6}$ & Prototype/engineering model tested in relevant environment \\
\hline $\mathbf{7}$ & Engineering model/similar equipment tested in space \\
\hline $\mathbf{8}$ & Full operational capability \\
\hline
\end{tabular}

After an extensive debate to allocate every method with a certain $T R L$, where it was taken into account past missions, experiments and literature publications, the TRL $f$ vector became (Table 7.5):
Table 7.5: $\quad T R L f$ for the different deviation strategies.

\begin{tabular}{|l|c|}
\hline \multicolumn{1}{|c|}{ Strategies } & TRL $\boldsymbol{f}$ \\
\hline Nuclear Impactor & 0.5 \\
\hline Kinetic Impactor & 1 \\
\hline Solar Collector & 0.375 \\
\hline Mass Driver & 0.375 \\
\hline Electric Propulsion & 0.5 \\
\hline
\end{tabular}

Despite the fact that no mission to test this technology is flying, Kinetic Impactor technology has been considered to be fully developed, TRL 8 . Examples of this technology are many and even a deflection mission with scheduled launch in 2011, called Don Quijote.

Nuclear and Low-Thrust technology has been considered to be in intermediate stage where the critical function and characteristics has been demonstrated. The main reason is because even when the basic technology is completely tested the environment in which would be used would be completely different from what was designed and tested.

Finally, Solar Collector and Mass Drivers are believed to be in a TRL 3. Basic experiments with the technology to be used have been already performed, examples are deployment of inflatable structures, autonomous drilling of surfaces, etc.

The warning time $\left(t_{w}\right)$ is divided by the TRLf to account for the required time to develop a certain technology. Tables 7.6, 7.7 and 7.8 must be handled carefully, since these results are the outcome of stretching the $t_{w}$ dimension.

Table 7.6: $\quad$ Nyx $T R L$ table (a=1.93 A.U.).

\begin{tabular}{|c|c|c|c|c|}
\hline & Nuke & Impactor & Solar & MD \\
\hline Impactor & 0 & & & \\
\hline Solar M & 100 & 0 & & \\
\hline MD & 100 & 0 & 85.9 & \\
\hline $\mathrm{LT}$ & 100 & 0 & 82.3 & 82.3 \\
\hline
\end{tabular}

Table 7.7: $\quad$ Castalia $T R L$ table (a=1.06 A.U.).

\begin{tabular}{c|c|c|c|c|}
\multicolumn{1}{c}{} & \multicolumn{1}{c}{ Nuke } & \multicolumn{1}{c}{ Impactor } & Solar & MD \\
\cline { 2 - 5 } Impactor & 45.6 & & & \\
\cline { 2 - 5 } Solar M & 97.2 & -45.44 & & \\
\cline { 2 - 5 } MD & 100 & -45.5 & 100 & \\
\cline { 2 - 5 } LT & 88.4 & 41.4 & 87.6 & 87.6 \\
\cline { 2 - 5 } & & &
\end{tabular}


Table 7.8: $\quad$ 1999KW4 TRL table (a=0.64 A.U.)

\begin{tabular}{c|cc|c|c|}
\multicolumn{1}{c}{} & Nuke & \multicolumn{1}{c}{ Impactor } & Solar & MD \\
\cline { 2 - 5 } Impactor & 0 & & & \\
\cline { 2 - 5 } Solar M & 78.7 & 0 & & \\
\cline { 2 - 5 } MD & 100 & 0 & 100 & \\
\cline { 2 - 5 } LT & 100 & 43.2 & 71.1 & 71.1 \\
\cline { 2 - 5 } & & &
\end{tabular}

The first conclusion from the TRLf dominance is that Kinetic Impactor cannot be disposed as a reliable method. When Technology Readiness is considered the Kinetic Impactor Pareto front covers parts of the domain that the other strategies are not able to cover, this is obviously as a result of the $t_{w}$ stretching.

Maybe, the most important conclusion from the $T R L f$ calculation is that Kinetic Impactor dominates clearly over Low-Thrust.

\section{FINAL REMARKS}

In this work, different strategies to deviate dangerous NEO have been modelled and compared. A set of analytical formulas have been proposed to compute the variation of the MOID due to a given deviation strategy. The variation has been expressed as a function of the warning time and the wet mass of the spacecraft at the Earth.

The comparison has been performed on three classes of asteroids, by means of a multi-criteria optimisation. The sets of Pareto optimal solutions for each strategy have been compared by defining the dominance of one Pareto set over another. Moreover a technology readiness factor has been introduced in order to estimate the actual required warning time.

This preliminary comparison demonstrated how the nuclear blast and the solar concentrators are generally dominant if the TRL is not taken into account. On the other hand if the TRL is considered the Kinetic Impactor becomes competitive.

A more accurate comparison would require an improvement of all the models as well as to take into account a possible fragmentation of the asteroid due to a too aggressive action.

\section{REFERENCES}

[1] Naja G., "The Second Report by ESA's Long-Term Space Policy Committee (LSPC)", Directorate of Strategy and Technical Assessment, ESA, Paris, May 1999.

[2] Don Quijote Homepage: http://www.esa.int/gsp/completed/neo/donquijote.html.

[3] Deep Impact Homepage: http://deepimpact.jpl.nasa.gov

[4] NEAR mission Homepage: http://near.jhuapl.edu
[5] Rosetta

mission http://www.esa.int/esaMI/Rosetta

Homepage:

[6] Tedeschi W. J., Remo J. L., Schulze J. F., Young R. P., "Experimental Hypervelocity Impact Effects on Simulated Planetesimal Materials", International Journal of Impact Engineering, Vol. 17, Elsevier Science Ltd, 1995, pp. 837-848.

[7] McInnes C. R., "Deflection of near-Earth asteroids by kinetic energy impacts from retrograde orbits", Planetary and Space Science, Vol. 52, Elsevier, 2004, pp. 587-590.

[8] Ivashkin V. v., Smirnov V. V., "An analysis of some methods of asteroid hazard mitigation for the Earth", Planetary Space Science, Vol. 43, No. 6, Elsevier Science Ltd, 1995, pp. 821-825.

[9] Spitale J. N., "Asteroid Hazard Mitigation Using the Yarkovsky Effect”, Science, Vol. 295, 5 April 2002, pp. 77.

[10] Melosh H. J., Nemchinov I. V., Zetzer Y. I., "Nonnuclear strategies for deflecting comets and asteroids" in Gehrels T. (editor), Hazard Due to Comets and Asteroids, University of Arizona Press, Tucson, 1994, pp. 1111- 1132 .

[11] Lu E. T., Love S. G., "Gravitational tractor for towing asteroids", Nature, Vol. 438, 2005, pp. 177-178.

[12] Hall C. D., Ross I. M., "Dynamic control problems in the deflection of near-Earth objects", AAS 97-640, 1997.

[13] Canavan G. H., Solem J. C., Rather J. D. G. (editors), Proceeding of the Near-Earth-Object Interception Workshop, Los Alamos National Laboratory, 1992.

[14] Bourdoux A., Izzo D., "Characterization and hazard mitigation of resonant returning Near Earth Objects", Final Stage Report, ACT internal report: ACT-RPT4100-AB-CHMRRNEO05.

[15] Izzo D., "On the deflection of Potentially Hazardous Objects" 15th AAS/AIAA Space Flight Mechanics Conference, AAS 05-141, Copper Mountain, Colorado, 2005.

[16] Carusi A., Valsecchi G. B., D'abramo G., Bottini A., "Deflecting NEOs in Route of Collision with the Earth", Icarus, Vol. 159, 2002, pp. 417-422.

[17] Scheeres D. J., Schweickart R. L., "The Mechanics of Moving Asteroids", 2004 Planetary Defense Conference: Protecting Earth from Asteroids, 23-26 February 2004, Orange Country, California.

[18] Conway B. A., "Near-Optimal Defection of EarthApproaching Asteroids", Journal of Guidance, Control and Dynamics, Vol. 24, No. 5, 2001, pp. 1035-1037.

[19] Park S.-Y., Ross I. M., "Two-Body Optimization for Deflecting Earth-Crossing Asteroids", Journal of Guidance, Control and Dynamics, Vol. 22, No. 3, 1999, pp. 995-1002.

[20] Ross I. M., Park S.-Y., Porter S. D. V., "Gravitational Effects of Earth in Optimizing $\Delta \mathrm{v}$ for Deflecting EarthCrossing Asteroids", Journal of Spacecraft and Rockets, Vol. 38, No. 5, September-October 2001, pp. 759-764.

[21] Schaub H., Junkins J. L., Analytical mechanics of space systems, AIAA Education Series, AIAA, 2003, pp. $592-623$. 
[22] Battin R. H., An Introduction to the Mathematics and Methods of Astrodynamics, Revised Edition, AIAA Education Series, AIAA, 1999.

[23] Wang J., Davis A. M., Clayton R. N., Hashimoto A., "Evaporation of Single Crystal Forsterite: Evaporation Kinetics, Magnesium Isotope Fractionation, and Implications of Mass-dependent Isotopic Fractional of a Diffusion-Controled Reservoir", Geochimica et Cosmochimica Acta, Vol. 63, No. 6, 1999, pp. 953-966.

[24] Kahle R., Kührt E., Hahn G., Knollenberg J., "Physical Limits of Solar Collectors in Deflecting Earththreatening Asteroids", Aerospace Science and Technology, Vol. 10, 2006, pp. 256-263.

[25] Thomas M., "Inflatable Space Structures", IEEE Potentials Magazine, 1992, pp. 29-32.

[26] Wertz J. R., Larson W. J., Space Mission Analysis and Design, Third ed., Microcosm Press 2003.

[27] Olds J., Charania A., Graham M., Wallace J., "The League of Extraordinary Machines: A rapid and Scalable Approach to Planetary defense Against Asteroid Impactors", 2004.

[28] Ahrens T. J., Harris A. W., "Deflection and Fragmentation of Near-Earth Asteroids," Nature, Vol. 360, 1992, pp. 429-433.

[29] Remo J. L., "Energy Requirements and Payload Masses for Near-Earth Objects Hazard Mitigation," Acta Astronautica, Vol. 47, No. 1, 2000, pp. 35-50.

[30] Smith P. L., Barrera M. J., Campbell E. T., Fedman K. A., Peterson G. E., Smith G. N., "Deflecting a Near Earth Object with Today's Space Technology," 2004 Planetary Defense Conference: Protecting Earth from Asteroids, 2004.

[31] Vasile M., De Pascale P., "On the Preliminary Design of Multiple Gravity-Assist Trajectories".

[32] Vasile M., "A Hybrid Multi-Agent Collaborative Search Applied to the Solution of Space Mission Design Problems", Proceeding of Global Optimisation, 2005, pp. 1-11.

[33] Carusi A., Valsecchi G. B., Greenberg R., "Planetary Close Encounters: Geometry of Approach and Postencounter Orbital Parameters", Celestial Mechanics and Dynamical Astronomy, Vol. 49, 1990, pp. 111-131.

[34] Milani A., Chesley S. R., Chodas P. W., Valsecchi G. B., "Asteroids Close Approaches: Analysis and Potential Impact Deflection”, in Asteroid III, Bottke W. F. Jr. et al. eds., University of Arizona, Tucson, pp. 5569.

[35] Conway B. A., "Optimal Low-Thrust Interception of Earth-Crossing Asteroids", Journal of Guidance, Control and Dynamics, Vol. 20, No. 5, 1997, pp. 9951002.

[36] Izzo D., Bourdoux A., Walker R., Ongaro F., “Optimal Trajectories for the Impulsive Deflection of Near Earth Objects", 56th International Astronautical Congress, IAC-05-C1.5.06.

[37] Bonanno C., "An analytical approximation for the MOID and its consequences", Astronomy and Astrophysics, Vol. 360, 2000, pp. 411-416.

[38] Kahle R., Hahn G., Kührt E., "Optimal deflection of NEOs en route of collision with the Earth", Icarus, Vol. 182, 2006, pp. 482-488.
[39] Flury W., Häntschel G., Grizner C., Fasoulas S., Altenburger L., NEO-MIPA Near-Earth Object Hazard Mitigation Publication Analysis, final report, 2001.

[40] Valsecchi G. B., Milani A., Gronchi G. F., Chesley S. R., "Resonant returns to close approaches: Analytical theory", Astronomy and Astrophysics, 2003, pp. 11791196. 